\title{
Human Immunodeficiency Virus (HIV)-Induced Neurotoxicity: Roles for the NMDA Receptor Subtypes 2A and $2 \mathrm{~B}$ and the Calcium-Activated Protease Calpain by a CSF-derived HIV-1 Strain
}

\author{
Lauren A. 0'Donnell, ${ }^{1}$ Arpita Agrawal, ${ }^{1}$ Kelly L. Jordan-Sciutto, ${ }^{2}$ Marc A. Dichter, ${ }^{1}$ David R. Lynch, ${ }^{1}$ and \\ Dennis L. Kolson ${ }^{1}$ \\ ${ }^{1}$ Department of Neurology, School of Medicine, and ${ }^{2}$ Department of Pathology, School of Dental Medicine, University of Pennsylvania, Philadelphia, \\ Pennsylvania 19104
}

\begin{abstract}
Neuronal damage in human immunodeficiency virus type 1 (HIV-1) infection in the brain is thought to occur at least in part through NMDA receptor (NMDAR) excitation initiated by soluble neurotoxins from HIV-infected brain macrophages. Furthermore, brain regions enriched in NMDAR-2A (NR2A) and NMDAR-2B (NR2B) subunits, such as the hippocampus, are particularly vulnerable. Using cultured rat hippocampal cells and HIV-1-infected human monocyte-derived macrophages (HIV/MDM), we examined the role of NR2A and NR2B in HIV/MDM-induced hippocampal neuronal death. We used the primary HIV-1 strain Jago derived from the CSF of an individual with HIV-associated dementia and that robustly replicates in MDM. We found the following: (1) hippocampal neuronal susceptibility to HIV/MDM excitotoxins varies according to the developmental expression patterns of NR2A and NR2B; (2) NMDAR activation by HIV/MDM results in neuronal calpain activation, which results in neuronal death; and (3) selective antagonists of homomeric NR2B/NR2B- and heteromeric NR2A/NR2B-containing NMDARs, as well as an inhibitor of calpain activity, afford neuroprotection against HIV/MDM. These studies establish a clear link between macrophage HIV infection, neuronal NR2A and NR2B activation, and calpain-mediated hippocampal neuronal death. They further suggest a dominant role for NR2A and NR2B in determining neuronal susceptibility in HIV-infected brain. Antagonists of NR2A and NR2B subunits as well as inhibitors of calpain activation offer attractive neuroprotective approaches against HIV in both developing and mature brain.
\end{abstract}

Key words: HIV-1; NMDA receptor; calpain; hippocampus; virus; neurotoxicity; macrophage

\section{Introduction}

Infection of the brain with human immunodeficiency virus type 1 (HIV-1) is associated with neuronal dendritic damage, apoptosis, and necrosis, all of which are thought to result from release of neurotoxins from HIV-infected macrophages (HIV/MDM) and other activated glial cells (Dawson et al., 1993; Gelbard et al., 1995; Petito and Roberts, 1995; Adamson et al., 1996; Kaul et al., 2001). HIV-1 productively infects cells of the macrophage lineage (macrophages and microglia) in vitro and in vivo (Wiley et al., 1986; Cosenza et al., 2002; Fischer-Smith et al., 2004). This infection results in release of proinflammatory cytokines, chemokines, and excitatory amino acids, which can kill and injure neurons

Received Aug. 25, 2005; revised Dec. 1, 2005; accepted Dec. 6, 2005.

This work was supported by National Institutes of Health Grants NS45956 (D.R.L.), NS043994, and NS27405 (D.L.K). L.A.O. was supported by the University of Pennsylvania Training Grant T32 Al07632 in HIV Pathogenesis and Penn Center for AIDS Research Grant P30 Al045008. We thank Margaret Maronski for preparation of hippocampal cultures.

Correspondence should be addressed to Dr. Dennis L. Kolson, Department of Neurology, University of Pennsylvania, 280C Clinical Research Building, 415 Curie Boulevard, Philadelphia, PA 19104. E-mail: kolsond@mail. med.upenn.edu.

DOI:10.1523/JNEUROSCI.4617-05.2006

Copyright $\odot 2006$ Society for Neuroscience $\quad$ 0270-6474/06/260981-10\$15.00/0
(Tardieu et al., 1992; Power et al., 1998; Chen et al., 2002). Brain regions such as the hippocampus, basal ganglia, and forebrain, which are susceptible to NMDAR-mediated excitotoxicity, are particularly vulnerable in HIV infection as well (Masliah et al., 1992; Petito et al., 2001; Archibald et al., 2004; Sa et al., 2004), and in vitro neuronal death caused by HIV/MDM can be effectively blocked by NMDA receptor (NMDAR) antagonists (Giulian et al., 1990; Lipton, 1993; Kaul et al., 2001). These observations suggest a role for NMDARs in the neurodegeneration induced by HIV-1 infection, and they have prompted clinical HIV neuroprotection trials with NMDAR antagonists (Lipton, 2004; Yiannoutsos et al., 2004).

Functional NMDARs are heteromeric assemblies of four subunits: two NR1 subunits and two NR2 subunits, of which there are four types (NR2A, NR2B, NR2C, and NR2D) (Lynch and Guttmann, 2002; Waxman and Lynch, 2005). NR1 subunits bind glycine, whereas NR2 subunits bind glutamate and quinolinic acid. Although all four NR2 subunits bind glutamate with equal affinity, NR2A and NR2B trigger greater excitotoxicity than NR2C and NR2D (for review, see Lynch and Guttmann, 2002; Waxman and Lynch, 2005). Interestingly, brain regions enriched in NMDAR composed of NR2A and NR2B subunits (hippocam- 
pus, basal ganglia, and forebrain) are commonly injured in excitotoxic insults (ischemia and epilepsy) and also in HIV-1 infection (Conti et al., 1999; Everall et al., 1999; Heyes et al., 2001; Lynch and Guttmann, 2002; Archibald et al., 2004; Sa et al., 2004; Waxman and Lynch, 2005). Furthermore, regions such as the cerebellum that express relatively high levels of NR2C and low levels of NR2B are commonly spared in both (Lynch and Guttmann, 2002). These observations suggest a common pathway of neuronal death mediated by NR2A and/or NR2B subunits in classic excitotoxic brain injury as well as in HIV-1 infection. Accordingly, the development of NR2A-selective (Liu et al., 2004) and NR2B-selective (Lynch and Guttmann, 2001) antagonists has been driven by a widespread interest in producing nontoxic, selective NMDAR antagonists as neuroprotectants against such brain injury (Lipton, 2004).

We hypothesized that neurons expressing NR2A- and NR2B-containing receptors would be particularly susceptible to neurotoxicity induced by exposure to HIV/MDM. Therefore, to define the role of NR2A and NR2B in HIV/MDMinduced neuronal death, we examined the responses of developing cultured neonatal rat hippocampal neurons to culture supernatants from HIV/MDM. Embryonic rat hippocampal neurons demonstrate temporally varying expression of NR2A- and $\mathrm{NR} 2 \mathrm{~B}$-containing receptors and offer an excellent model system for studying NMDAR-mediated excitotoxicity. With our system, we validated previous reports of release of excitotoxins from MDM through HIV-1 infection (Giulian et al., 1990; Brew et al., 1995; Xiong et al., 2000). We then demonstrated that hippocampal neuronal susceptibility to HIV/MDM excitotoxins is predicted by the appearance of NR2A- and NR2B-containing NMDA receptors. Finally, we found that such NMDAR activation by HIV/MDM results in neuronal calpain activation, which leads to neuronal death. Because NR2A and NR2B expression vary both developmentally and regionally within the brain, our observations suggest a significant role for NR2A and NR2B in determining agerelated and regional neuronal susceptibility to HIV-induced damage in both the pediatric and adult brain.

\section{Materials and Methods}

Chemicals and reagents. Glutamate, MK801 [(+)-5-methyl-10,11dihydro-5H-dibenzo [a,d] cyclohepten-5,10-imine maleate], AP-5, ifenprodil, and Ro25-6981 [R-( $R, S)-\alpha$-(4-hydroxyphenyl)- $\beta$-methyl-4(phenylmethyl)-1-piperidine propranol] were purchased from Tocris

B mock, respectively).
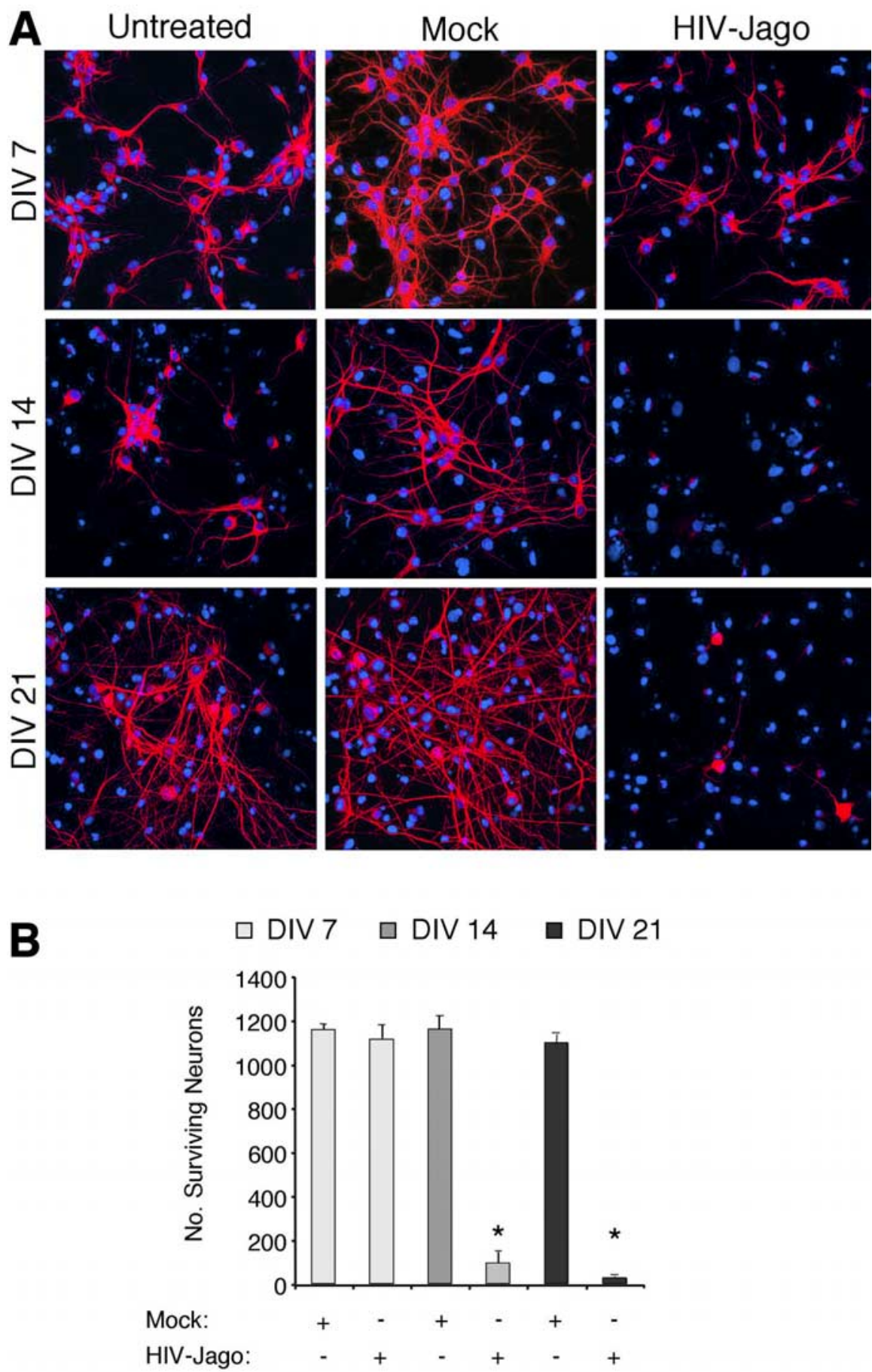

Figure 1. Hippocampal neuronal susceptibility to neurotoxins produced by HIV-1-infected macrophages is age dependent. Supernatants from mock-infected or HIV-Jago-infected macrophages were applied to rodent hippocampal neurons that had been cultured for DIV 7, DIV 14, or DIV 21 on glass coverslips. Twenty-four hours later, cultures were stained for MAP-2 ( $A$, red) and for nuclei (Hoechst 33342; blue). Magnification for confocal micrographs is $400 \times$. $\boldsymbol{B}$, Neuronal survival as determined by counts of MAP-2-reactive cells. Data represent the average \pm SD counts of multiple coverslips $\left(n=6 ;{ }^{*} p<0.01\right.$ vs DIV 14 mock or DIV 21

Cookson (Ellisville, MO). Proteinase-K, zinc, the calpain inhibitor MDL28170 (carbobenzoxy-valinyl-phenylalaninal), and an anti-microtubule associated protein-2 (MAP-2) monoclonal were from Sigma (St. Louis, MO). Conantokin-G was purchased from Bachem (King of Prussia, PA). An anti-NMDA receptor-1 monoclonal antibody (catalog \#556308) was purchased from BD Transduction Laboratories (Lexington, KY), and an anti-NMDA receptor 2A (catalog \#07-632) antibody was from Upstate Biotechnology (Lake Placid, NY). An anti-NMDA re- 
ceptor 2B antibody (catalog \#718600; directed against the N-terminal 251 amino acids) was from Zymed (San Francisco, CA). Antibody A38, which is directed against calpain-cleaved spectrin, was described previously (Roberts-Lewis et al., 1994) and was a generous gift from Dr. Robert Siman (University of Pennsylvania, Philadelphia, PA). An antiglyceraldehyde-3-phosphate dehydrogenase (GAPDH) antibody (sc20357) was purchased from Santa Cruz Biotechnology (Santa Cruz, CA), an anti-neurofilament polyclonal (NB300-135) was purchased from Novus Biologicals (Littleton, CO), and an anti- $\beta$-tubulin III monoclonal (MU177) was purchased from BioGenex (San Ramon, CA). NVPAAM077 [ $R$ )-[( $S)$-1-(4-bromo-phenyl)-ethylamino]-(2,3-dioxo1,2,3,4-tetrahydro-quinoxalin-5-yl)-methyl]phosphonic acid] was a kind gift from Dr. Yves Auberson at Novartis Institutes for BioMedical Science (Basel, Switzerland). Efavirenz was provided by DuPont Pharmaceuticals (Wilmington, DE).

Preparation of MDM and HIV infections. MDM were isolated from primary blood mononuclear cells from healthy volunteers as described previously (Chen et al., 2002). Cells were collected in accordance with protocols approved by the University of Pennsylvania Committee on Studies Involving Human Beings. Cells were cultured in six-well plates $\left(1.25 \times 10^{6}\right.$ cells per well) for $7 \mathrm{~d}$ in macrophage media (DMEM with $10 \%$ fetal bovine serum, $10 \%$ horse serum, $1 \%$ penicillin/streptomycin, and $1 \%$ nonessential amino acids) supplemented with macrophage colony stimulating factor $(100 \mathrm{U} / \mathrm{ml})$. Macrophage media was changed on day 7 in each well, inoculated with equivalent amounts of cell-free HIV-1 inoculum (100 ng of p24 per well), and incubated for $18 \mathrm{~h}$. Cells were washed twice with PBS and incubated in fresh macrophage media. Macrophage supernatants were monitored for $\mathrm{p} 24$ antigen content by ELISA (NEN, Boston, MA). In each case, HIV infection of macrophages was confirmed by serial quantification of p24 antigen levels in the culture supernatants over time. Supernatants were used for application to neuronal cultures only when productive infection was confirmed (p24 values were typically $100-400 \mathrm{pg} / \mathrm{ml}$ supernatant). In control macrophage cultures, efavirenz, a non-nucleoside reverse transcriptase inhibitor that is highly effective in suppressing HIV replication in cells of the macrophage lineage, was added (20 nM) $1 \mathrm{~h}$ before HIV inoculation. Supernatants from HIV/MDM and non-infected macrophages (mock/MDM) were collected at selected time points after infection and stored at $-80^{\circ} \mathrm{C}$.

HIV-1 isolate Jago is a macrophage-tropic isolate derived from cellfree CSF from a patient with confirmed HIV-associated dementia (Chen et al., 2002). Stocks of Jago were prepared in primary T-lymphocytes derived from the blood of healthy volunteers through the University of Pennsylvania Center for AIDS Research Virology Core. As for other macrophage-tropic HIV strains, Jago productively infects primary T-lymphocytes and macrophages but not transformed T-cell lines (Chen et al., 2002).

Preparation of primary neuronal cultures. Primary rat hippocampal cultures were prepared from embryonic day 17 Sprague Dawley rat pups as described previously (Wilcox et al., 1994). Cells were plated at a density of $2 \times 10^{5}$ cells per $35 \mathrm{~mm}$ dish on glass coverslips precoated with poly-L-lysine (Peninsula Laboratories, San Carlos, CA) and maintained in neurobasal media with $\mathrm{B} 27$ supplement at $37^{\circ} \mathrm{C}, 5 \% \mathrm{CO}_{2}$. Half of the medium volume was replaced every $7 \mathrm{~d}$, and cultures were used at 7,14 , or $21 \mathrm{~d}$ in vitro (DIV).

Quantification of HIV-induced neurotoxicity. Neuronal cultures were exposed to HIV/MDM and mock/MDM supernatants at a final dilution of $1: 10$ for $24 \mathrm{~h}$ at $37^{\circ} \mathrm{C}, 5 \% \mathrm{CO}_{2}$. Cultures were washed once with PBS and fixed in $4 \%$ paraformaldehyde/4\% sucrose in PBS for $1 \mathrm{~h}$, followed by permeabilizing in $100 \%$ methanol ( $10 \mathrm{~min}$ ) and $0.2 \%$ Triton $\mathrm{X}-100 /$ PBS (10 min). Cultures were then washed twice with PBS, blocked with $10 \%$ goat serum in PBS (45 min at room temperature), and exposed to anti-MAP-2 antibody ( $60 \mu \mathrm{M}$ in $10 \%$ goat serum in PBS) for $1 \mathrm{~h}$ at room temperature. After washing, a tetramethylrhodamine isothiocyanateconjugated goat anti-IgG secondary antibody (66 $\mu \mathrm{M} ; 45 \mathrm{~min}$ at room temperature) was used for detection of MAP-2 antibody binding. The number of surviving MAP-2-labeled neurons was estimated by blinded counting of all fields $(\sim 40$ at $400 \times)$ in two adjacent vertical columns through the center of each coverslip, proceeding from top $\rightarrow$ bottom $\rightarrow$ top. For each condition, five to six coverslips were counted from two or
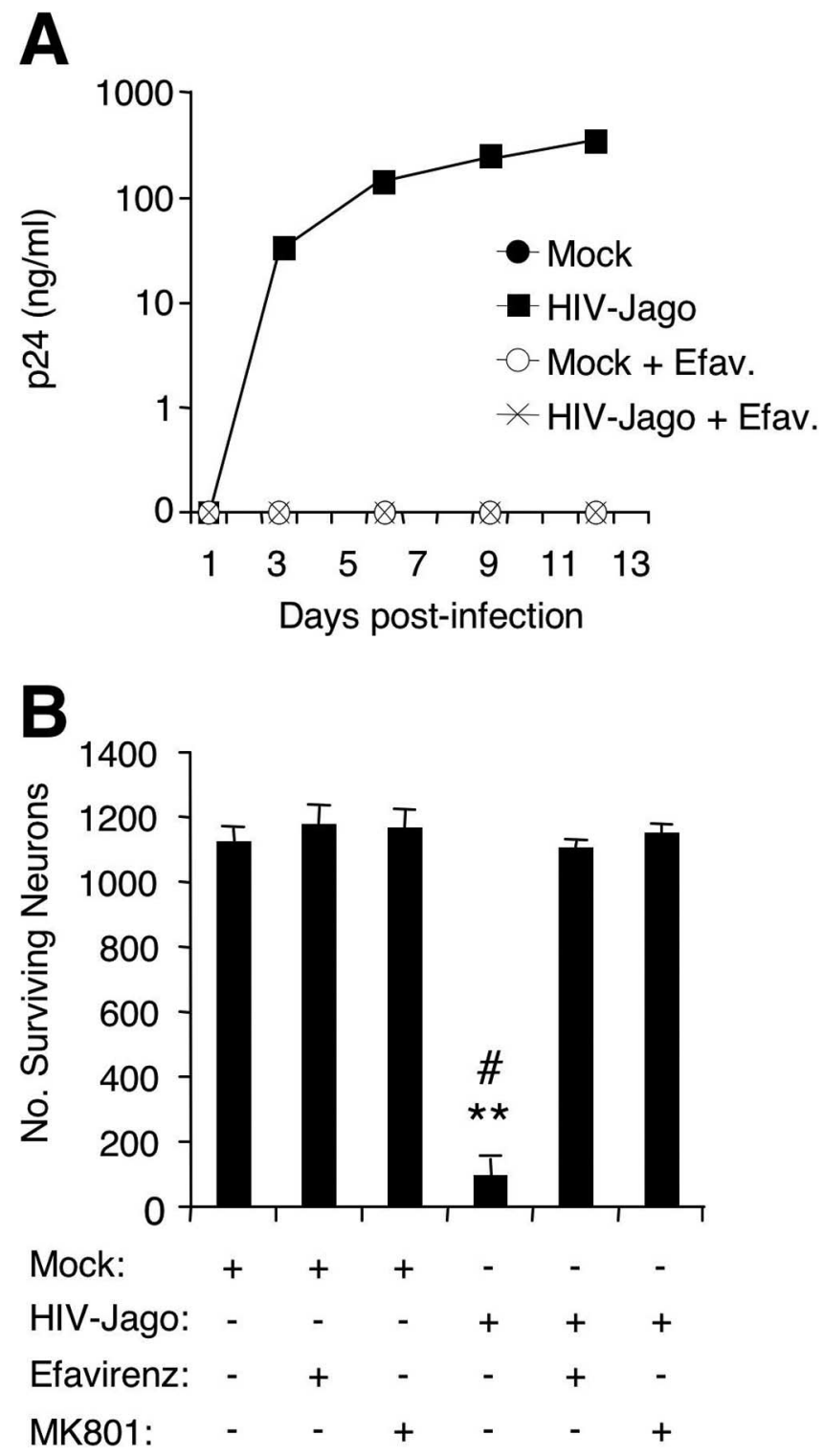

Figure 2. MDM release excitotoxins during productive HIV-1 infection. HIV-1 infection of MDM (indicated by supernatant p24 antigen content; $y$-axis) was blocked with the nonnucleoside reverse transcriptase inhibitor efavirenz $(\boldsymbol{A})$, and supernatants were added to DIV 21 hippocampal neurons $(\boldsymbol{B})$. Control hippocampal cultures received mock-infected supernatants with or without efavirenz. The number of surviving neurons was determined as in Figure $1 B$. The NMDA receptor antagonist MK801 $(10 \mu \mathrm{M})$ was added to hippocampal cultures $1 \mathrm{~h}$ before addition of macrophage supernatants ( ${ }^{* *} p<0.01$ vs HIV-Jago/efavirenz; ${ }^{\#} p<0.01$ vs HIVJago plus MK801).

more independent experiments, and the average \pm SE number of surviving neurons was determined.

Filtration, heat-inactivation, protease digestion, and glutamate pyruvate transaminase treatment of HIV/MDM supernatants. MDM supernatants were centrifuged through Centriplus filters (Millipore, Bedford, MA) (molecular weight cutoffs 50,10 , or $3 \mathrm{kDa}$ ) for $2 \mathrm{~h}$ at $2800 \times \mathrm{g}$, and filtrates $(<50,<10$, or $<3 \mathrm{kDa}$ fractions) were collected. The portion of supernatants retained by the filter $(>3 \mathrm{kDa}$ fraction) was resuspended in a volume of macrophage medium equal to the filtrate volume. For heat inactivation, supernatants were boiled for $10 \mathrm{~min}$ and cooled to $37^{\circ} \mathrm{C}$ before testing for neurotoxicity. For protease digestion, supernatants were filtered through a $3 \mathrm{kDa}$ filter and then incubated with proteinase- $\mathrm{K}$ $\left(1 \mu \mathrm{g} / \mathrm{ml}\right.$ ) for $2 \mathrm{~h}$ at $37^{\circ} \mathrm{C}$. The enzyme was removed from the supernatants by filtration through a $3 \mathrm{kDa}$ filter. Glutamate was removed from supernatants and from media spiked with glutamate by addition of glu- 
tamate pyruvate transaminase (GPT) $(100 \mu \mathrm{g} /$ $\mathrm{ml}$; Sigma), pyridoxal-L-phosphate $(100 \mu \mathrm{M})$, and pyruvate $(10 \mathrm{~mm})$ for $1 \mathrm{~h}$ at $37^{\circ} \mathrm{C}$.

Treatments with NMDA receptor antagonists and calpain inhibitors. Stock solutions of MK801, AP-5, ifenprodil, Ro25-6981, and Conantokin-G were made at $10 \mathrm{~mm}$ in water, zinc was reconstituted at $5 \mathrm{~mm}$ in water, and NVPAAM077 was reconstituted at $5 \mathrm{~mm}$ in 1 equivalent $\mathrm{NaOH}$ in water. Stocks of MDL28170 were made at $100 \mathrm{~mm}$ in DMSO. MK801, ifenprodil, Ro25-6981, and MDL28170 were used at a final concentration of $10 \mu \mathrm{M}, \mathrm{AP}-5$ was used at $100 \mu \mathrm{M}$, Conantokin-G was used at $6 \mu \mathrm{M}$, and zinc and NVP-AAM077 were used at 500 nM. NMDA receptor antagonists were added to cultures $1 \mathrm{~h}$ before the addition of MDM supernatants or NMDA receptor agonists. Calpain antagonists were added to cells incubated in HEPES-buffered physiological salt solution for $1 \mathrm{~h}$, and then HIV/ MDM or mock/MDM supernatants were applied to the cultures with the antagonists present.

Western blotting. Cell cultures were washed twice with cold PBS and lysed in ice-cold Laemmli buffer (0.625 м Tris-HCl, pH 6.8, 2\% SDS, and $10 \%$ glycerol) containing a protease inhibitor mixture (Sigma). Lysates were boiled for $5 \mathrm{~min}$, and equivalent amounts $(20 \mu \mathrm{g})$ of lysate were loaded onto $10 \%$ polyacrylamide gels $(7 \%$ when blotting with NMDA receptor antibodies) and subjected to SDS-PAGE. Gels were blotted on to Immunoblot polyvinylidene difluoride membrane (Bio-Rad, Hercules, CA) and blocked in PBS with $0.05 \%$ Tween-20 (PBS-Tween) and 5\% nonfat milk for $1 \mathrm{~h}$ at room temperature. Primary antibodies were incubated overnight at $4^{\circ} \mathrm{C}$ in blocking solution. All primary antibodies were used at 1:1000 with the exception of antibody A38 (rabbit anticalpain cleaved spectrin), which was used at 1:10,000. Membranes were washed three times with PBS-Tween and incubated in secondary antibody conjugated to HRP (125 ng/ml; Cell Signaling Technology, Beverly, MA) in PBSTween with $5 \%$ nonfat milk for $1 \mathrm{~h}$ at room temperature. Membranes were washed three times with PBS-Tween, developed in LumiGLO chemiluminescent substrate (Cell Signaling Technology), and exposed to Basic Autorad film. For densitometric analysis, films were scanned into Adobe Photoshop (Adobe Systems, San Jose, CA), and a fixed cursor area centered over each band was assessed for pixel density, with normalization to GAPDH signal.

\section{Results}

Hippocampal neurons demonstrate age-dependent susceptibility to

HIV-infected macrophages

To determine how a vulnerable neuronal cell type succumbs to neurotoxicity from HIV-1-infected macrophages, we used a model system of developing fetal rat hippocampal mixed neuronal/glial cultures exposed to culture supernatants harvested from HIV/MDM. Because NR2B/NR2B- and NR2A/ NR2B-containing receptors predominate within the hippocampus and because their expression varies with developmental age (Monyer et al., 1994), we first determined the age-dependent susceptibility of developing hippocampal neurons to HIV/MDM.

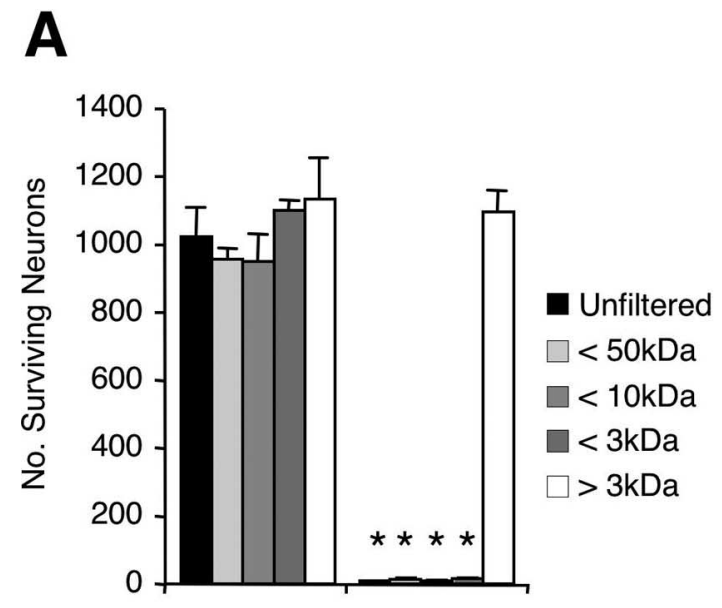

B
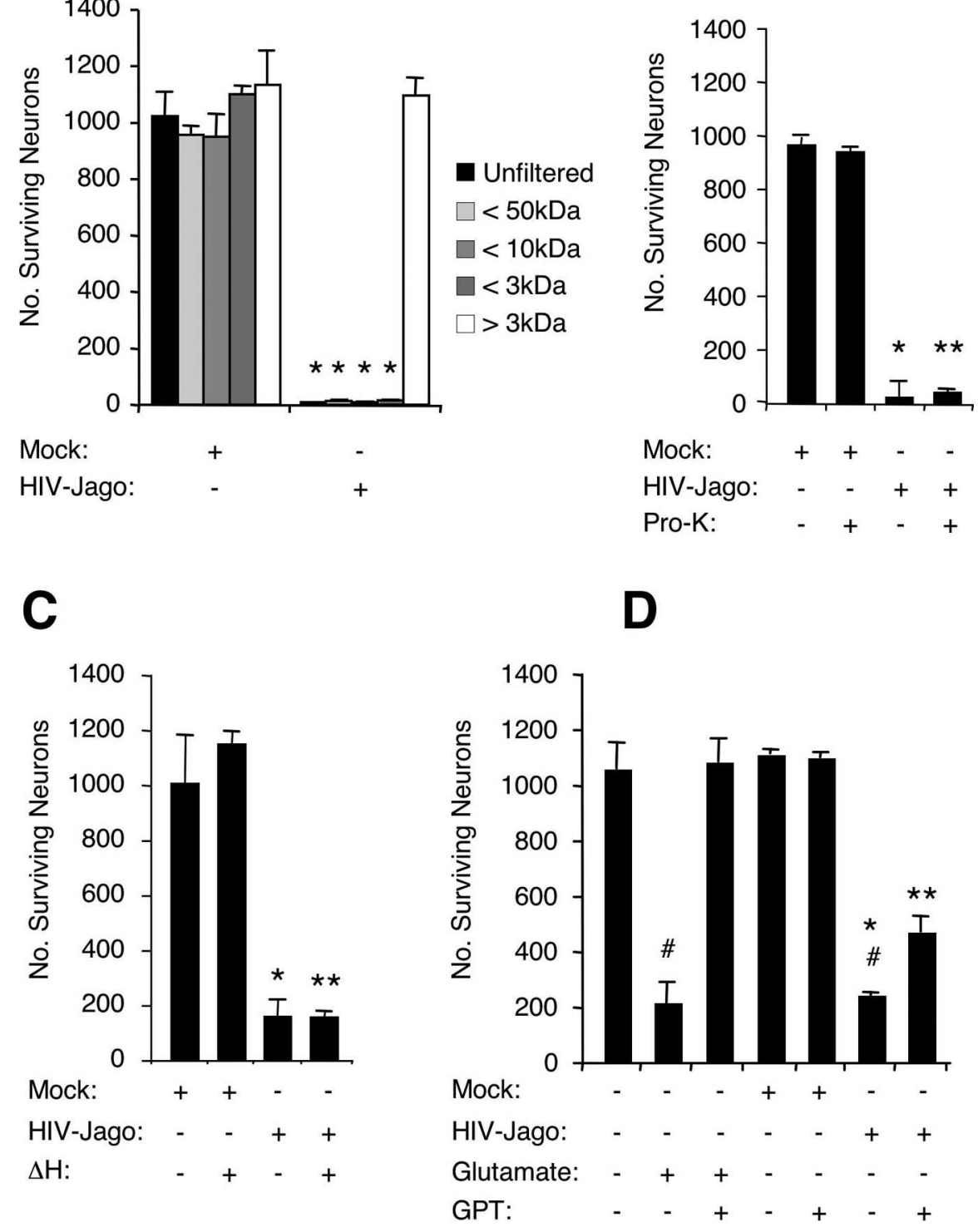

Figure 3. Glutamate is partially responsible for HIV/MDM neurotoxicity. Supernatants were filtered through membranes with molecular weight cutoffs of $50 \mathrm{kDa}(<50 \mathrm{kDa}), 10 \mathrm{kDa}(<10 \mathrm{kDa})$, and $3 \mathrm{kDa}(<3 \mathrm{kDa})$ and assayed for neurotoxicity on DIV 21 hippocampal cultures $(\boldsymbol{A})$. The neurotoxicity of factors larger than $3 \mathrm{kDa}$ in the supernatants $(>3 \mathrm{kDa})$ was determined by resuspending the filter retentate in macrophage media in the same volume as the filtrate. $\boldsymbol{B}$, Supernatants were exposed to proteinase- $\mathrm{K}\left(1 \mu \mathrm{g} / \mathrm{ml}\right.$ for $2 \mathrm{~h}$ at $\left.37^{\circ} \mathrm{C}\right)$, and then treated supernatants were filtered through a $3 \mathrm{kDa}$ filter before testing for neurotoxicity on DIV 21 hippocampal cultures. C, Supernatants were boiled (10 $\mathrm{min} ; \Delta \mathrm{H})$, cooled to $37^{\circ} \mathrm{C}$, and tested for neurotoxicity on DIV 21 hippocampal cultures. Neither proteinase-K treatment nor boiling abrogated neurotoxicity $\left({ }^{*} p<0.01\right.$ vs mock; ${ }^{* *} p<0.01$ vs mock treated). $\boldsymbol{D}$, The contribution of glutamate from HIV-Jago supernatants was assayed by enzymatic degradation of glutamate in supernatants by treatment with GPT. Control media was spiked with $100 \mu \mathrm{m}$ glutamate alone. Control and MDM supernatants (mock and HIV-Jago) were treated with GPT, added to DIV 21 hippocampal cultures (1:10 dilution for $24 \mathrm{~h}$ for a final dilution of $10 \mu \mathrm{m}$ glutamate), and followed by MAP-2 staining and cell counting ( ${ }^{*} p<0.01$ vs GPT treated).

We infected macrophages with the macrophage-tropic, CNSderived HIV-1 strain Jago (Chen et al., 2002) and subsequently exposed rodent hippocampal neuronal cultures of different ages (DIV 7, DIV 14, and DIV 21) to the HIV/MDM culture supernatants (Fig. $1 A$ ). After $24 \mathrm{~h}$, we stained the cultures for MAP-2 expression (Fig. $1 A$ ) and quantified the number of surviving neurons (Fig. $1 B$ ). As shown in Figure 1, after exposure to HIV/ MDM (Jago) supernatants, DIV 7 neuronal cultures showed no neuronal loss, whereas profound neuronal loss was observed at DIV 14 and DIV 21 compared with cultures exposed to superna- 
A $\quad \square$ DIV $7 \quad \square$ DIV $14 \quad \square$ DIV 21

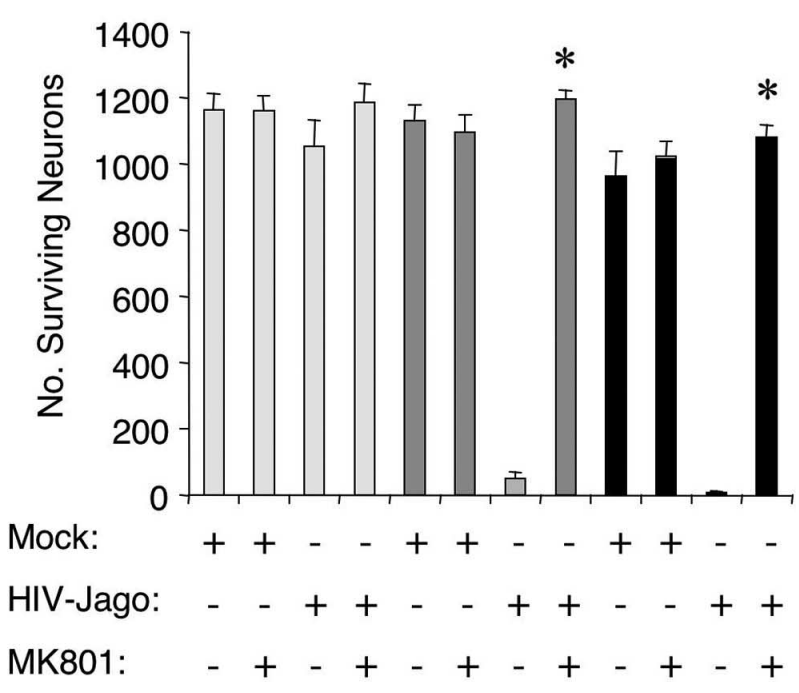

B
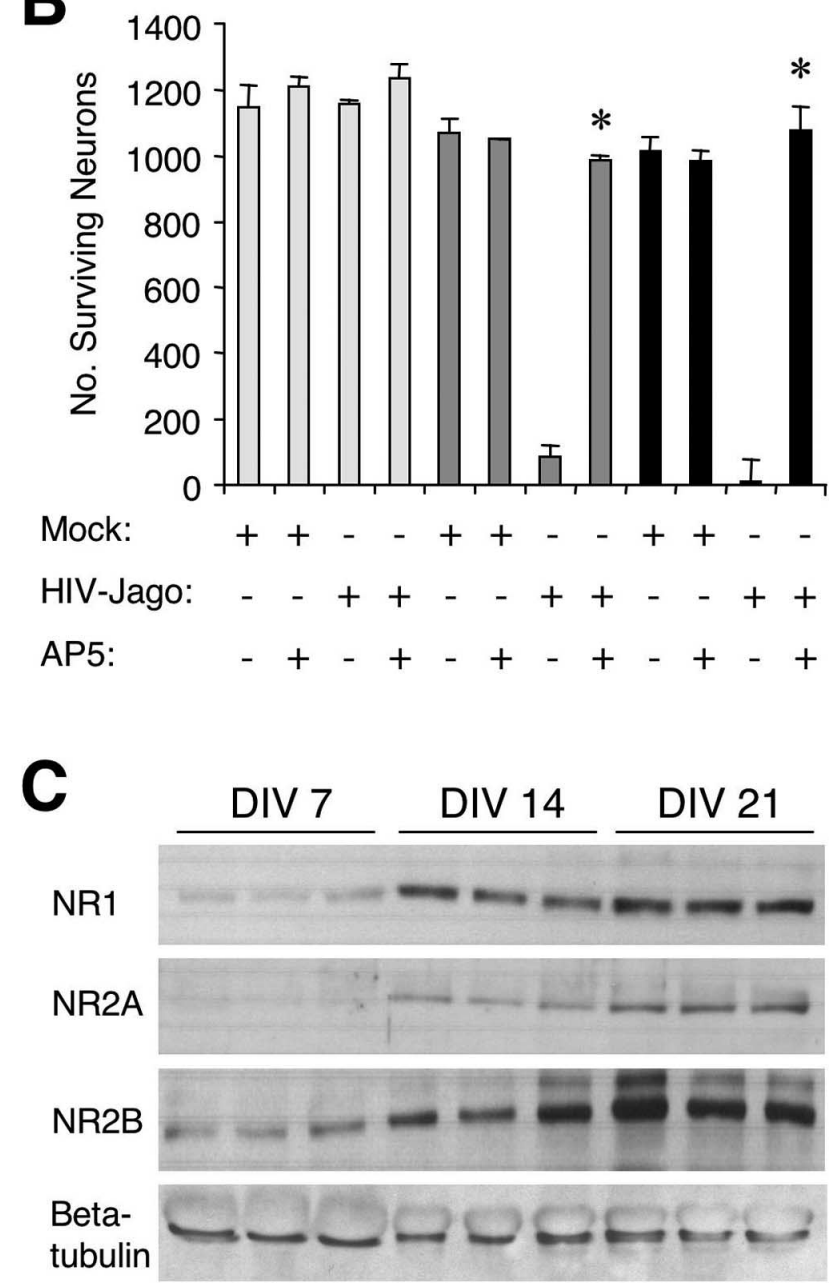

Figure 4. Age-dependent susceptibility of developing hippocampal neurons to HIV/MDM neurotoxicity is predicted by NMDA receptor expression. Hippocampal cultures were maintained for DIV 7, DIV 14, or DIV 21 and preincubated with the noncompetitive NMDA receptor antagonist MK801 $(10 \mu \mathrm{m} ; \boldsymbol{A})$ or the competitive NMDA receptor antagonist AP-5 $(100 \mu \mathrm{m} ; \boldsymbol{B})$ for $1 \mathrm{~h}$ before exposure to MDM supernatants. Cultures were incubated for $24 \mathrm{~h}$ and assayed for the number of surviving neurons ( ${ }^{*} p<0.01$ same day HIV-Jago). $C$, Western blots for NMDA receptor subunits (NR1, NR2A, and NR2B) and for $\beta$-tubulin III as a protein loading control.
Table 1. Subunit specificity of NMDA receptor antagonists

\begin{tabular}{lll}
\hline Antagonist & Subunit bound & NMDAR inhibited \\
\hline Ifenprodil & NR2B & NR2B/2B \\
Ro25-6981 & NR2B & NR2B/2B \\
Conantokin-G & NR2B & NR2B/2B, NR2A/2B \\
Zinc & NR2A & NR2A/2A \\
NVP-AAM077 & NR2A & NR2A/2B, NR2A/2A
\end{tabular}

NMDA receptor antagonists used in Figure 5. The NMDA receptor subunit to which each antagonist directly binds and the NMDA receptor subunit combinations that are inhibited by each antagonist are listed (for review, see Waxman and Lynch, 2005). $<1$. $>$

tants from mock-infected macrophages. Inhibition of HIV-1 replication in the macrophages with the non-nucleoside reverse transcriptase inhibitor efavirenz (Fig. $2 \mathrm{~A}$ ) prevented the neurotoxicity (Fig. $2 B$ ), indicating that HIV-1 replication within the macrophages is necessary for the release of neurotoxins. In addition, the NMDAR antagonist MK801 was protective (Fig. 2C), confirming that NMDAR activation mediates this HIV/MDM neurotoxicity.

\section{Glutamate partially contributes to}

HIV/MDM-induced neurotoxicity

Consistent with previous reports (Giulian et al., 1996) describing the presence of low-molecular-weight excitotoxins in the HIV/ MDM supernatants, ultrafiltration (Fig. $3 A$, cutoff of 50,10 , or 3 $\mathrm{kDa}$ ), proteinase-K treatment (Fig. $3 B$ ), and boiling of the macrophage supernatants (Fig. $3 C$ ) before application to neuronal cultures failed to abrogate the neurotoxicity. To address the possibility that glutamate released from the HIV/MDM was primarily responsible for the toxicity (Jiang et al., 2001), we treated supernatants with GPT, which lowers glutamate concentrations through transamination of glutamate, before exposure to neurons. As shown in Figure 3D, GPT treatment completely blocked toxicity induced by $10 \mu \mathrm{m}$ glutamate and showed significant but partial protection against toxicity induced by HIV/MDM supernatants. This suggests that HIV/MDM excitotoxins, including glutamate, are the major source of HIV/MDM neurotoxicity.

Age-related hippocampal neuronal susceptibility to HIV-infected macrophages is predicted by NMDAR-2B and NMDAR-2A expression

Because hippocampal cultures demonstrate age-dependent susceptibility to HIV/MDM (Fig. 1) and because NMDAR subunit expression and function vary with developmental age in hippocampal neurons, we determined the ability of specific NMDAR antagonists to block HIV/MDM neurotoxicity in hippocampal neurons maintained in culture for various ages. As shown in Figure 4, noncompetitive [MK801 (Fig. 4A)] and competitive [AP-5 (Fig. 4B)] NMDAR antagonists each inhibited neurotoxicity at DIV 14 and DIV $21(n=6 ; p<0.01)$. Furthermore, when examined by Western blotting (Fig. 4C), NMDAR expression was barely detectable in DIV 7 cultures, whereas DIV 14 and DIV 21 cultures showed increasing expression of NMDAR subunits NR1, NR2A, and NR2B. Specifically, expression of both NR2A and NR2B subunits increased dramatically from DIV 7 through DIV 21, whereas NR1 expression increased dramatically between DIV 7 and DIV 14 but remained relatively constant between DIV 14 and DIV 21.

\section{NMDAR-2B and NMDAR-2A antagonists protect} hippocampal neurons against HIV/MDM toxicity We hypothesized that expression of functional NR2B/NR2Bcontaining receptors would account for the susceptibility of DIV 
14 neurons to HIV/MDM supernatants. Furthermore, although NR2B was detected at each age, NR2A appeared only weakly by DIV 14 and more strongly at DIV 21. We expected that NR2A- and NR2B-selective antagonists (Table 1) would differentially protect these neurons at different times in culture. Using two noncompetitive NR2B-selective antagonists, ifenprodil and Ro25-6981 (Lynch and Guttmann, 2001), we found that DIV 14 cultures were completely protected, whereas DIV 21 cultures were only partially protected (Fig. $5 A, B$ ). Although each of these agents binds to the NR2B receptor, each is effective only in inhibiting NMDAR function in the context of NR2B/ NR2B homomeric associations and not NR2A/NR2B heteromeric associations (Chenard and Menniti, 1999; Lynch and Guttmann, 2001). We therefore hypothesized that DIV 21 neurons, which express both NR2B/NR2B- and NR2A/NR2Bcontaining receptors, would be fully protected only by agents that block both NR2A- and NR2B-containing subtypes. As expected, Conantokin-G, which blocks both NR2B/NR2B- and NR2A/NR2Bcontaining receptors (Klein et al., 2003), afforded complete protection for DIV 14 as well as DIV 21 neurons (Fig. 5C). In contrast, we found that zinc, a highaffinity antagonist of NR2A/NR2Acontaining receptors, provided no protection at either age (Fig. 5D). Finally, NVP-AAM077, which blocks both NR2A/ NR2A- and NR2A/NR2B-containing receptors (Liu et al., 2004), afforded no protection at DIV 14 and only partial protection at DIV 21 (Fig. 5E). These findings are summarized in Table 2. These observations strongly support the conclusion that age-dependent susceptibility of hippocampal neurons to HIV/MDM-induced excitotoxicity is mediated by NR2B/ NR2B- and NR2A/NR2B-containing receptor subtypes, and that blockade of both subtypes in mature neurons is essential for effective neuroprotection against HIV/MDM.

\section{NMDAR activation by HIV/MDM} results in activation of calpains One consequence of NMDAR activity is the activation of calpains, which are calcium-activated proteases that cleave cytoskeletal elements, such as spectrin, neurofilament, and MAP-2 (Goll et al., 2003), and functional elements, such as NMDAR-2B (Guttmann et al., 2001; Simpkins et al., 2003). We hypothesized that HIV/MDM supernatants would activate neuronal calpains, and that inhibition of such activation would prevent this neurotoxicity. As shown in Figure 6, exposure to HIV/

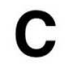

\section{7 IV 14 - DIV 21}
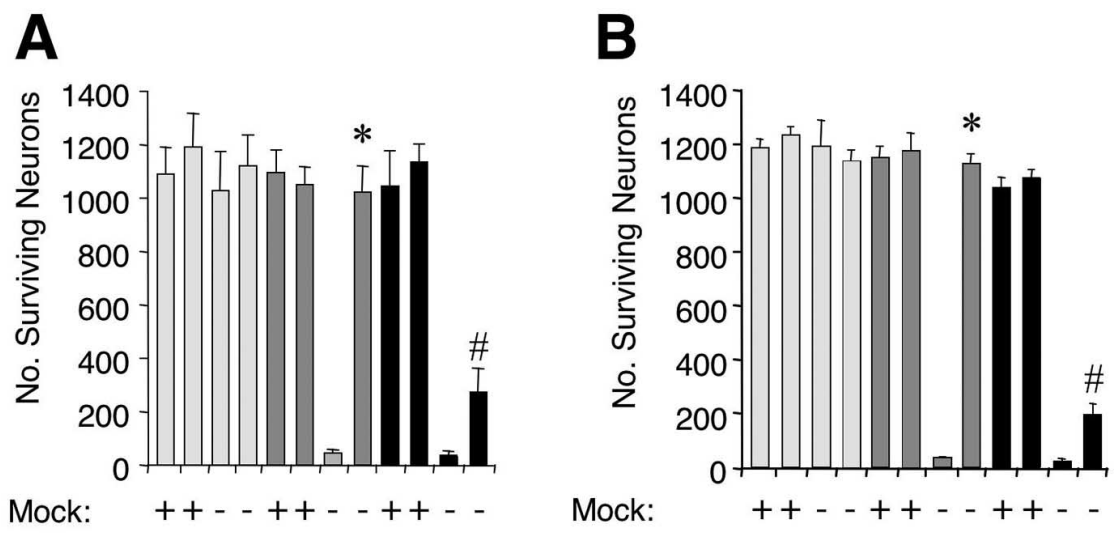

HIV-Jago: - - + + - - + + - + +

Ifenprodil: -+-+-+-+-+-+

HIV-Jago: $\quad--++--++-+++$

RO25-6981: - + - + + - + - + - +

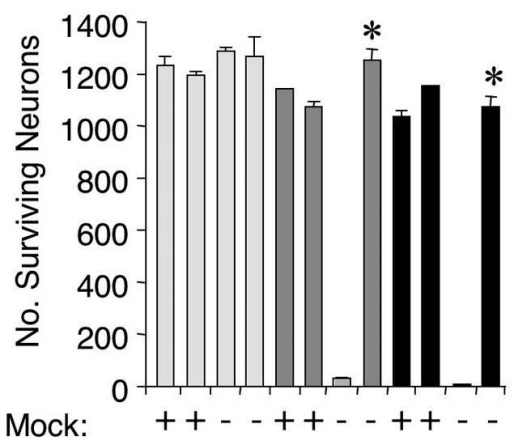

D
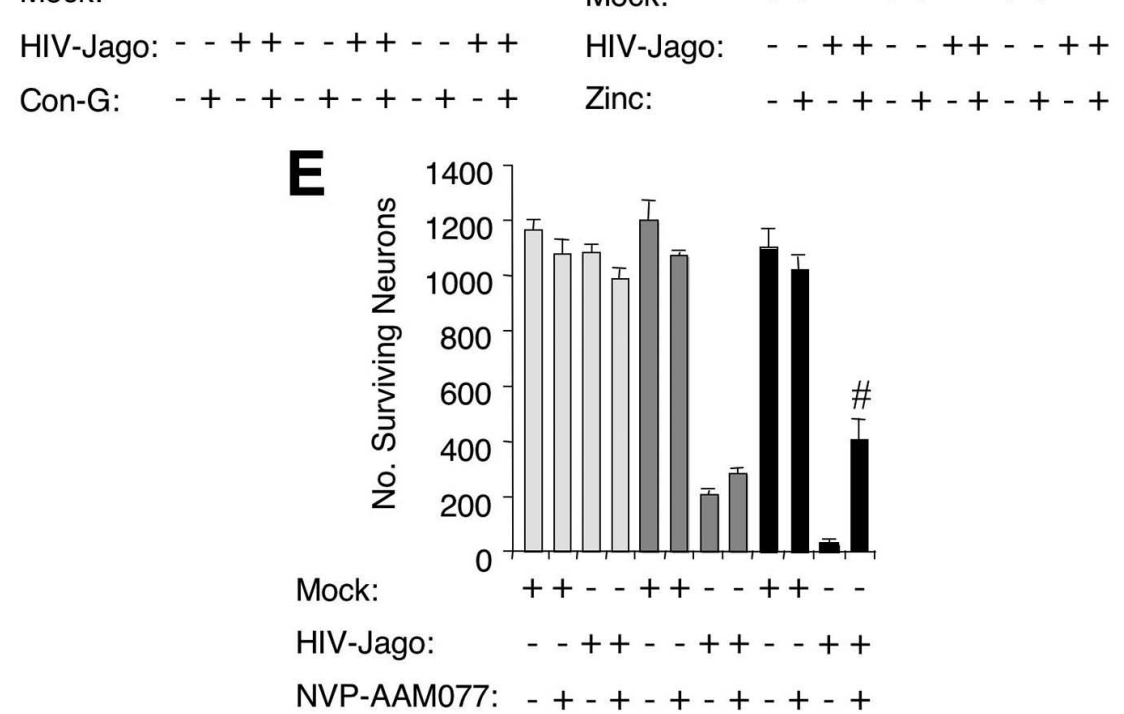

Figure 5. NMDAR subtype $2 B / 2 B$ - and $2 A / 2 B$ - selective antagonists protect hippocampal neurons from HIV/MDM neurotoxicity. Hippocampal cultures were preincubated for $1 \mathrm{~h}$ with subtype-specific NMDA receptor antagonists and then exposed to MDM supernatants for $24 \mathrm{~h}$. The number of surviving neurons was assayed by counting of MAP-2-reactive cells $\left({ }^{\#} p<0.05\right.$ vs same day HIV-Jago; ${ }^{*} p<$ 0.01 vs same day HIV-Jago.) Hippocampal cultures were exposed to the NR2B noncompetitive antagonists ifenprodil $(10 \mu \mathrm{m} ; \boldsymbol{A})$ Ro25-6981 (10 $\mu \mathrm{M} ; \boldsymbol{B})$. C, Conantokin-G, a competitiveNR2B antagonist that inhibits 2B/2B-and 2A/2B-containing NMDA receptors, was used at $6 \mu \mathrm{m}$. D, Zinc, a noncompetitive antagonist of NR2A at nanomolar concentrations, was used at $500 \mathrm{~nm}$. $\boldsymbol{E}$, NVP-AAM077, a competitive NR2A antagonist that inhibits NMDARs containing $2 \mathrm{~A} / 2 \mathrm{~A}$ or $2 \mathrm{~A} / 2 \mathrm{~B}$ subunits, was used at $500 \mathrm{~nm}$.

MDM supernatants resulted in a robust increase in calpain activity in hippocampal cultures (fourfold) (Fig. 6A), as evidenced by detection of calpain-specific spectrin cleavage products. Furthermore, calpain activation was prevented by preincu- 
Table 2. Summary of neuroprotection by NMDA receptor subunit-specific antagonists

\begin{tabular}{llc}
\hline & Neuroprotection & \\
\cline { 2 - 3 } Antagonist & DIV 14 & DIV 21 \\
\hline Ifenprodil & +++ & + \\
Ro25-6981 & +++ & + \\
Conantokin-G & +++ & +++ \\
Zinc & - & - \\
NVP-AAM077 & - & + \\
\hline
\end{tabular}

Neuroprotection against HIV/MDM by NMDA receptor antagonists was categorized as $75-100 \%$ protection $(+++), 50-75 \%$ protection $(++), 25-50 \%$ neuroprotection $(+)$, or $0-25 \%$ protection $(-)$ for DIV 14 or DIV 21 hippocampal neurons.

bation of neurons with MK801 (Fig. 6A). Moreover, we detected the characteristic spectrin-mediated NMDAR-2B cleavage pattern (Fig. 6C, open arrow), which was also prevented by preincubation with MK801. We confirmed that spectrin cleavage was the result of calpain activation by blocking the production of the cleavage product by preincubation of cells with the cellpermeable calpain inhibitor MDL28170 (Fig. 6E,F). These results thus clearly link HIV/MDM-associated NMDAR activation with neuronal calpain activation and subsequent cleavage of calpain-specific targets.

\section{Calpain activation contributes to HIV/MDM neurotoxicity}

Activity of calpains is often associated with cell death models of ischemia, spinal cord injury, and a range of neurodegenerative diseases, such as Alzheimer's disease and Huntington's disease (for review, see Goll et al., 2003). However, calpain inhibition is not universally neuroprotective because other neurodegenerative pathways can be activated in parallel with calpains (Ray et al., 2003; Yamashima, 2004). To determine whether calpain activation leads to HIV/MDM-induced hippocampal neuronal death, we determined the ability of the calpain inhibitor MDL28170 to prevent HIV/MDM-induced neurotoxicity. Figure 7 demonstrates a dose-dependent neuroprotective effect of MDL28170 up through the concentration $(10 \mu \mathrm{M})$ that we determined was effective in inhibiting calpain-mediated spectrin cleavage (Fig. 6). These results clearly show that calpain activation mediates cell death events downstream of NMDA receptor activation by exposure to HIV/MDM supernatants.

\section{Discussion}

We have demonstrated that the susceptibility of developing hippocampal neurons to HIV/MDM is determined by their expression of NMDAR-2A and NMDAR-2B subunits (NR2A and $\mathrm{NR} 2 \mathrm{~B}$, respectively), and that such toxicity is mediated at least in part by calpain activation. Effective neuroprotection in vitro is afforded by selective NR2A and NR2B antagonists and by inhibitors of calpain activation, and both receptor subtypes must be considered in implementing NMDAR-associated neuroprotection strategies. The results suggest that these agents, when used alone or in combination, could provide effective protection against neurodegeneration induced by HIV-1 infection in vivo. They further suggest that developmental and regional susceptibility to HIV-induced damage might be primarily determined by NMDAR subunit expression.

HIV infection of macrophages within the brain is thought to be a critical factor in triggering events leading to neuronal damage and death, and multiple studies in vitro and in vivo indicate that excitotoxicity mediated by activation of macrophages plays a major role (Brenneman et al., 1988; Heyes et al., 1989; Dreyer et al., 1990; Giulian et al., 1990; Tardieu et al., 1992; Dawson et al.,
1993; Lipton, 1993; Brew et al., 1995; Nottet et al., 1996; Power et al., 1998; Jiang et al., 2001; Kaul et al., 2001; Valle et al., 2004). Some of these models use HIV-infected monocytes/macrophages as a source of neurotoxins (Heyes et al., 1989; Giulian et al., 1990; Tardieu et al., 1992; Power et al., 1998), whereas others use application of recombinant proteins to neuronal cultures (Dreyer et al., 1990; Dawson et al., 1993; Lipton, 1993; Kaul et al., 2001) to model HIV/MDM neurotoxicity. In all, these studies have implicated a number of HIV- and MDM-associated neurotoxins that directly or indirectly activate neuronal NMDA receptors, including glutamate, quinolinic acid, platelet activating factor, reactive oxygen species, NTox, Tat, and gp 120. Our studies confirm some of these reports of low-molecular-weight heat- and proteaseresistant excitotoxins as major HIV/MDM-associated neurotoxins, which include glutamate (Fig. 3); however, our attempts to identify products of the kynurenine metabolic pathway (quinolinic acid) in macrophages as HIV/MDM excitotoxins (Brew et al., 1995; Nottet et al., 1996) in our system thus far are inconclusive (data not shown). Nonetheless, our NMDAR studies clearly indicate that neuronal NR2A and NR2B subunit expression patterns are a major determinant of hippocampal neuronal susceptibility to HIV/MDM-associated excitotoxins.

It is well established that NMDA receptors play a significant role in HIV-induced neurotoxicity in vitro (Dawson et al., 1993; Lipton, 1993; Power et al., 1998; Chen et al., 2002). However, the roles of NMDA receptors in vivo, and how NMDAR subtypes relate to regional and developmental neuronal susceptibility, remain essentially unexplored. We hypothesized that specific neuronal NMDAR subunit expression patterns would correlate with susceptibility to HIV/MDM in a vulnerable neuronal subtype. To address this question, we chose the rodent hippocampal culture system because it represents a well characterized model for studies of NMDAR-mediated excitotoxicity, and because the hippocampus is a region commonly damaged in HIV-infected brain. Consistent with the demonstrably low level of NMDAR-2A and NMDAR-2B subunit expression, DIV 7 hippocampal cultures showed no neurotoxicity during exposure to HIV/MDM supernatants, whereas marked toxicity was observed in DIV 14 and DIV 21 cultures. By DIV 14, these cultures exhibited robust NR1 and NR2B expression, suggesting the likely expression of functional NR2B/NR2B-homomeric receptors at this age. As predicted, we found that selective NR2B antagonists nearly completely protected DIV 14 cultures, thus confirming a role for the NR2B subunit in HIV/MDM-induced neurotoxicity in developing hippocampal neurons. Interestingly, Xiong et al. (2003) demonstrated that supernatants from HIV-infected macrophages could activate inward ionic currents in Xenopus oocytes expressing recombinant NR1/NR2B NMDA receptors, which is consistent with our observations of NR2B-mediated toxicity in DIV 14 cultures. Meeker et al. (2004) also demonstrated age-dependent susceptibility of rat neocortical neurons to CSF collected from HIV-infected individuals compared with CSF from HIVnegative individuals, but the role of NMDAR activation was not specifically addressed in that study.

Our observation that DIV 21 hippocampal neurons are only partially protected by NR2B/NR2B-selective antagonists is most straightforwardly explained by the appearance of NR2A subunits by this time and the formation of functional NR2A/NR2B heteromeric receptors, which are not blocked by noncompetitive NR2B antagonists. We found that the invertebrate neurotoxin Conantokin-G, which blocks not only these heteromeric receptors but also NR2B/NR2B-containing receptors (Klein et al., 2003), completely protected the neurons at DIV 21. The recently 
developed NR2A antagonist NVPAAM077, which can block both NR2A/ NR2A- and NR2A/NR2B-containing receptors (Liu et al., 2004), was also partially protective at DIV 21, consistent with our hypothesis that blocking abnormal activation of both subtypes of NMDARs is necessary for maximal neuroprotection against HIV/MDM. To our knowledge, this is the first demonstration of a neuroprotective effect of a selective NR2A antagonist, which may be vital for neuroprotection in the adult brain.

Our findings suggest that it is critical to consider the roles for both NR2A and NR2B subunits in neurotoxicity in vivo, because both contribute significantly to functional NMDAR in adult brain. In the developing brain, NR2B predominates (Conti et al., 1999; Waxman and Lynch, 2005). Because HIV-associated neuronal damage is particularly severe in the developing human brain and in adult brain regions that are highly enriched in NR2B expression (hippocampus, striatum, and frontal cortex), but not areas with little NR2B expression (cerebellum) (Masliah et al., 1992; Lynch and Guttmann, 2002; Archibald et al., 2004; Sa et al., 2004), it is interesting to speculate that such expression could at least partially account for selective neuronal damage in HIV-infected developing and mature brain.

One consequence of HIV/MDMinduced NMDAR activation in our hippocampal cultures is the activation of calpains, which clearly contributes to the observed neuronal death. Calpains are known to be activated in a number of excitotoxic insults, and inhibition of calpain activation has been demonstrated to protect neurons against such insults (for review, see Goll et al., 2003). We demonstrated calpain-dependent cleavage of the structural protein spectrin and of the NR2B subunit, which has only recently been defined as a target for calpain (Guttmann et al., 2001; Simpkins et al., 2003). Although the functional consequences of NR2B cleavage by calpain are not yet defined, calpain-cleaved NR2B-containing receptors remain on the surface of the neuron and might increase the susceptibility of the cell to excitotoxic injury (D. R. Lynch, unpublished observations). Interestingly, protection by the calpain inhibitor tested, MDL28170, was incomplete (Fig. 7), and this level of neuroprotection did not increase when we tested concentrations as high as $50 \mu \mathrm{M}$ (data not shown), which is beyond the concentrations typically used in cell protection studies (Simpkins et al., 2003; Brundel et al., 2004). One possible explanation for the incomplete protection by MDL28170 is that it only binds to cal-

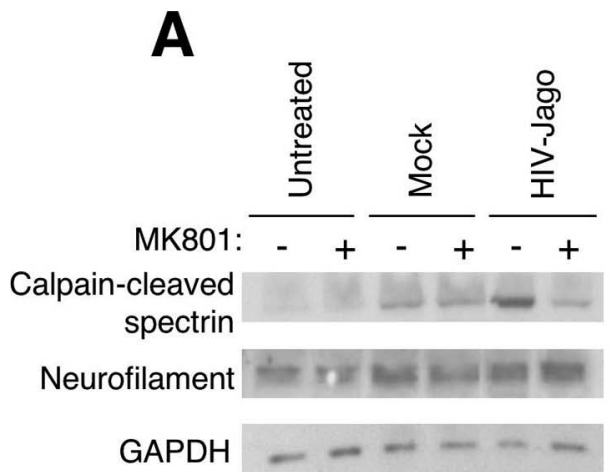

B
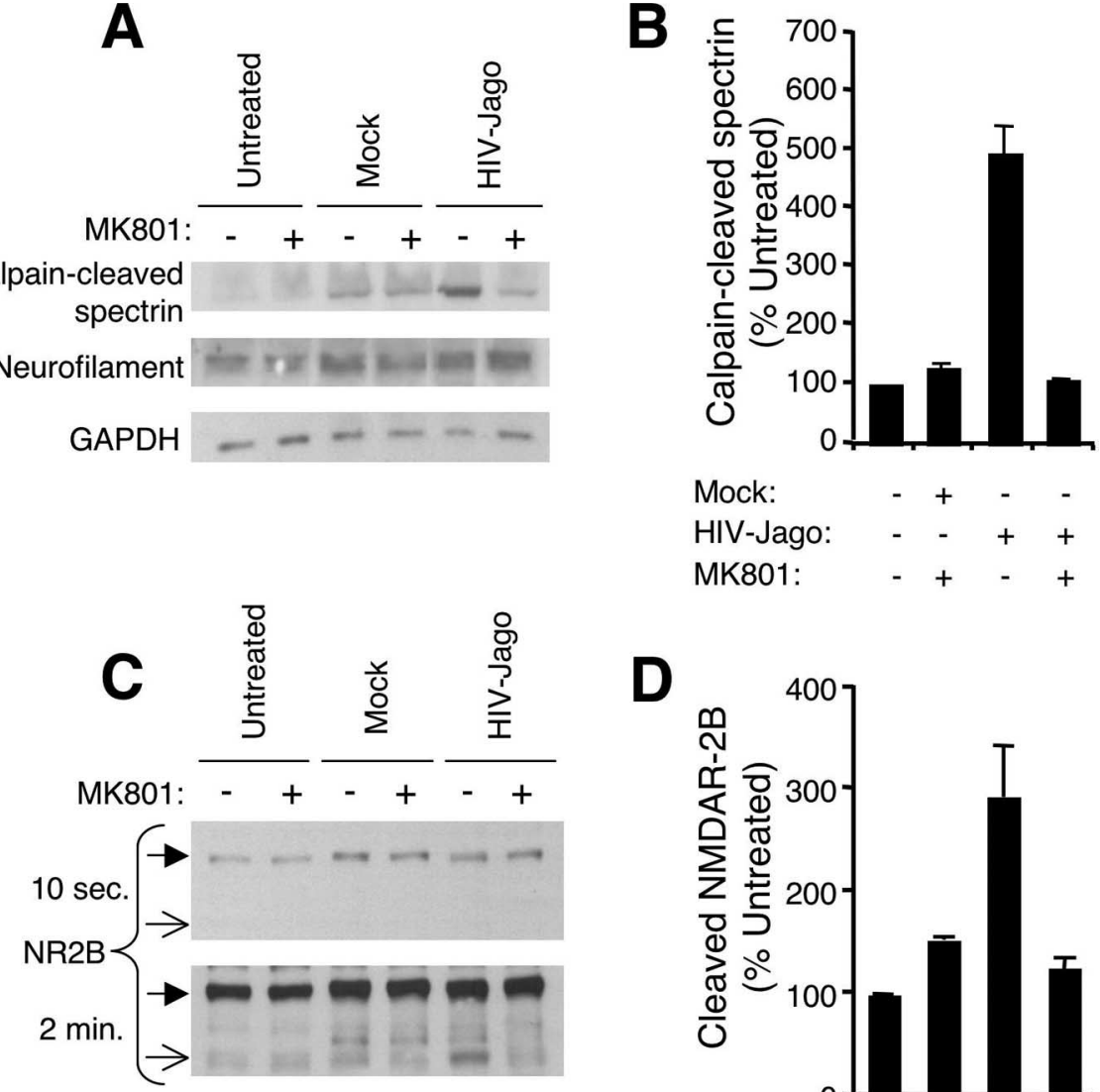

D
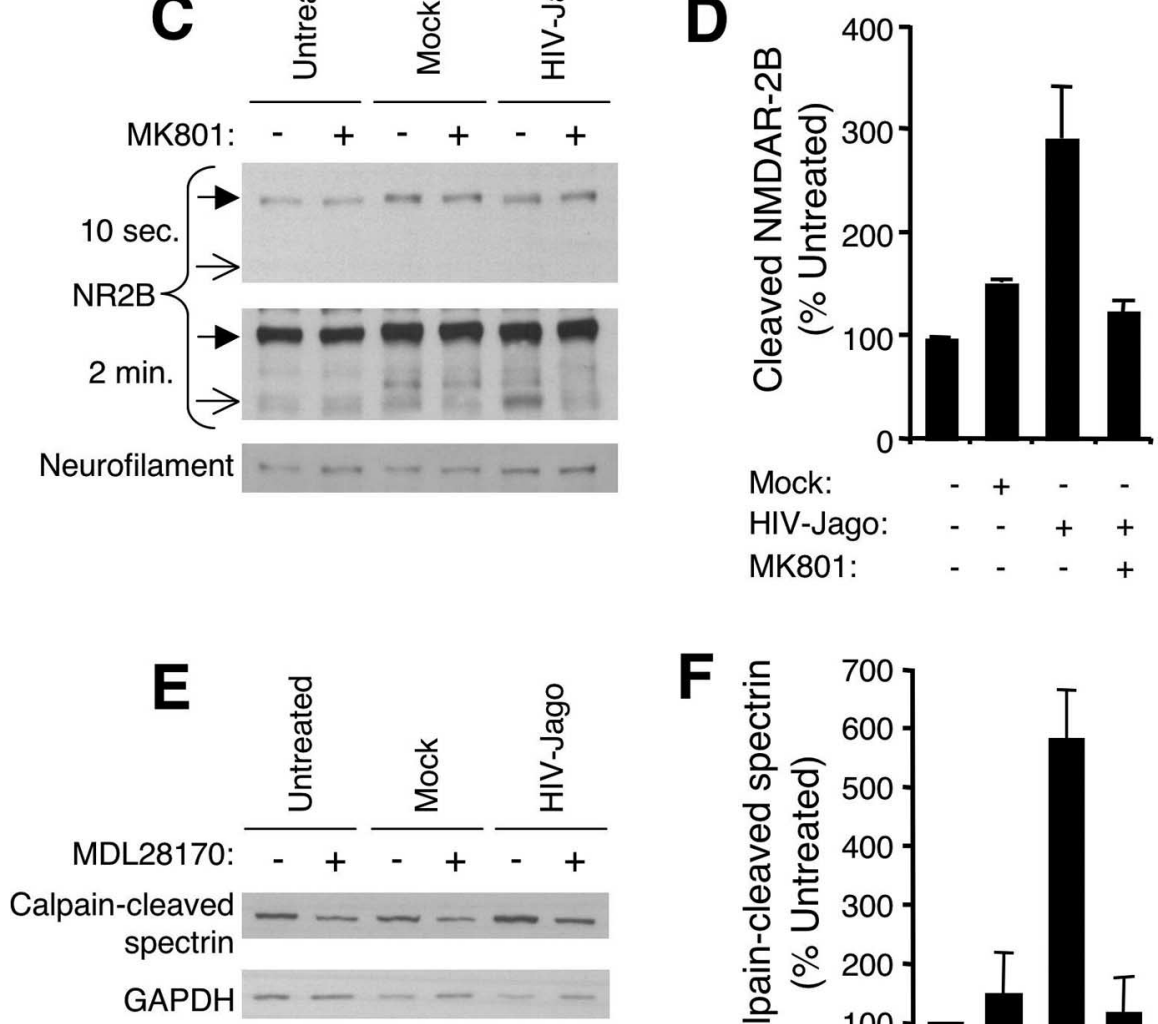

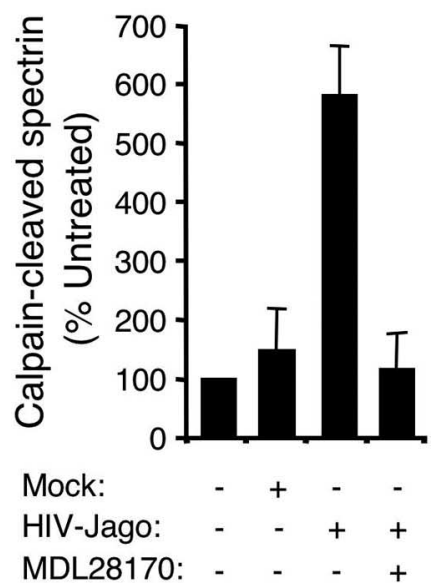

Figure 6. HIV/MDM activate calpains in an NMDA-receptor-dependent manner in hippocampal neurons. Hippocampal cultures were preincubated with MK801 $(10 \mu \mathrm{M})$ for $1 \mathrm{~h}$ before exposure to MDM supernatants. Neurons were exposed to supernatants for $1 \mathrm{~h}$ and then lysed for Western blot analysis. Band intensity was determined by densitometry (right panels). $\boldsymbol{A}$, Calpain activity was determined by detection of a calpain-dependent cleavage product of spectrin $(\boldsymbol{A}, \boldsymbol{B})$. Samples were also analyzed for another target of calpain cleavage, the NMDA receptor subunit NR2B $(\boldsymbol{C}, \boldsymbol{D})$. Two different exposure times ( $10 \mathrm{~s}$ and $2 \mathrm{~min}$ ) are depicted in $C$. The filled arrow corresponds to full-length NR2B, and the open arrow corresponds to the calpain-dependent cleavage product of NR2B. Cultures were also preincubated with the calpain inhibitor MDL21870 $(10 \mu \mathrm{M})$ before exposure to MDM supernatants and then analyzed for calpain-cleaved spectrin $(\boldsymbol{E}, \boldsymbol{F}) \cdot \boldsymbol{B}, \boldsymbol{D}$, and $\boldsymbol{F}$ represent densitometric analysis of blots.

pains after they have been bound and activated by calcium. Therefore, downstream death pathways might be activated before calpain inhibition is accomplished. Alternatively, MDL28170-resistant proteases such as caspases might also con- 


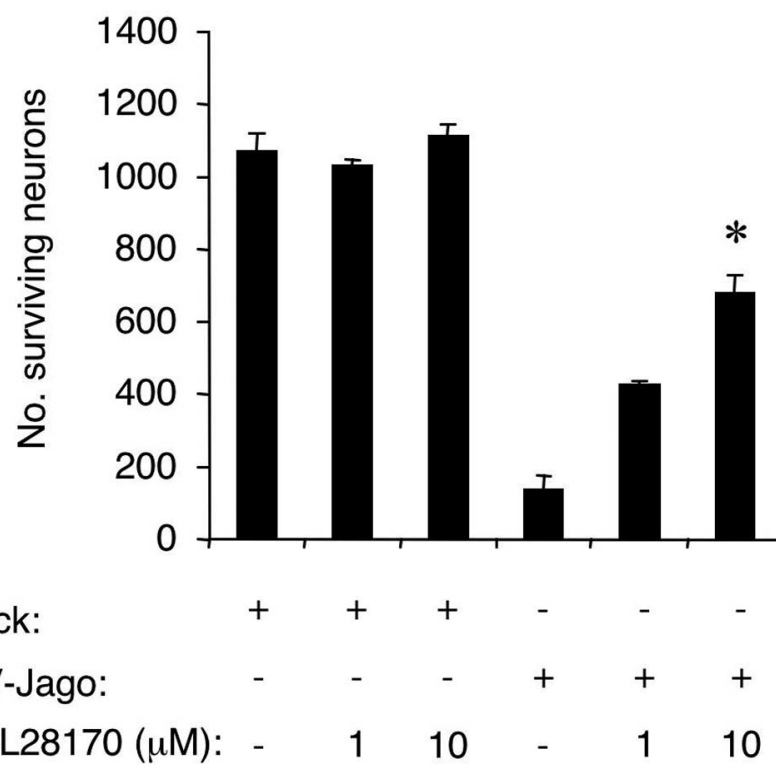

Figure 7. Calpains contribute to HIV/MDM-induced neurotoxicity. Hippocampal cultures were preincubated with the calpain inhibitor MDL28170 (1 or $10 \mu \mathrm{M})$ for $1 \mathrm{~h}$ before a $24 \mathrm{~h}$ exposure to mock and HIV-Jago supernatants and then assayed for the number of surviving neurons ( ${ }^{*} p<0.01$ vs HIV-Jago).

tribute significantly in this system (Ohagen et al., 1999; Garden et al., 2002). In addition, calpain activation was only observed in DIV 21 and DIV 14 cultures but not in DIV 7 cultures (data not shown), which suggests that calpain activation by HIV/MDM is a consequence of NMDA receptor activity in developing neurons.

Although it is clear that in vitro models of HIV-induced neurodegeneration cannot recapitulate all of the critical cell-cell interactions that determine neurotoxic effects of HIV replication in vivo, our infection model is consistent with neuropathological studies demonstrating HIV-associated neurodegeneration in brain regions particularly susceptible to excitotoxicity in both developing and adult brain. Whether the HIV-1 Jago strain is markedly neurovirulent because of higher levels of HIV/MDMassociated excitotoxins than other HIV-1 strains is of particular interest, and this is undergoing investigation in our laboratory. Our studies clearly suggest that neuronal NMDAR-2A and NMDAR-2B subunits as well as neuronal calpains offer attractive targets for neuroprotection against HIV infection in the brain.

\section{References}

Adamson DC, Wildemann B, Sasaki M, Glass JD, McArthur JC, Christov VI, Dawson TM, Dawson VL (1996) Immunologic NO synthase: elevation in severe AIDS dementia and induction by HIV-1 gp41. Science 274:1917-1921.

Archibald SL, Masliah E, Fennema-Notestine C, Marcotte TD, Ellis RJ, McCutchan JA, Heaton RK, Grant I, Mallory M, Miller A, Jernigan TL (2004) Correlation of in vivo neuroimaging abnormalities with postmortem human immunodeficiency virus encephalitis and dendritic loss. Arch Neurol 61:369-376.

Brenneman DE, Westbrook GL, Fitzgerald SP, Ennist DL, Elkins KL, Ruff MR, Pert CB (1988) Neuronal cell killing by the envelope protein of HIV and its prevention by vasoactive intestinal peptide. Nature 335:639-642.

Brew BJ, Corbeil J, Pemberton L, Evans L, Saito K, Penny R, Cooper DA, Heyes MP (1995) Quinolinic acid production is related to macrophage tropic isolates of HIV-1. J Neurovirol 1:369-374.

Brundel BJ, Kampinga HH, Henning RH (2004) Calpain inhibition prevents pacing-induced cellular remodeling in a HL-1 myocyte model for atrial fibrillation. Cardiovasc Res 62:521-528.

Chen W, Sulcove J, Frank I, Jaffer S, Ozdener H, Kolson DL (2002) Development of a human neuronal cell model for human immunodeficiency virus (HIV)-infected macrophage-induced neurotoxicity: apoptosis induced by HIV type 1 primary isolates and evidence for involvement of the Bcl-2/Bcl-xL-sensitive intrinsic apoptosis pathway. J Virol 76:9407-9419.

Chenard BL, Menniti FS (1999) Antagonists selective for NMDA receptors containing the NR2B subunit. Curr Pharm Des 5:381-404.

Conti F, Barbaresi P, Melone M, Ducati A (1999) Neuronal and glial localization of NR1 and NR2A/B subunits of the NMDA receptor in the human cerebral cortex. Cereb Cortex 9:110-120.

Cosenza MA, Zhao ML, Si Q, Lee SC (2002) Human brain parenchymal microglia express CD14 and CD45 and are productively infected by HIV-1 in HIV-1 encephalitis. Brain Pathol 12:442-455.

Dawson VL, Dawson TM, Uhl GR, Snyder SH (1993) Human immunodeficiency virus type 1 coat protein neurotoxicity mediated by nitric oxide in primary cortical cultures. Proc Natl Acad Sci USA 90:3256-3259.

Dreyer EB, Kaiser PK, Offermann JT, Lipton SA (1990) HIV-1 coat protein neurotoxicity prevented by calcium channel antagonists. Science 248:364-367.

Everall IP, Heaton RK, Marcotte TD, Ellis RJ, McCutchan JA, Atkinson JH, Grant I, Mallory M, Masliah E (1999) Cortical synaptic density is reduced in mild to moderate human immunodeficiency virus neurocognitive disorder. HNRC Group. HIV Neurobehavioral Research Center. Brain Pathol 9:209-217.

Fischer-Smith T, Croul S, Adeniyi A, Rybicka K, Morgello S, Khalili K, Rappaport J (2004) Macrophage/microglial accumulation and proliferating cell nuclear antigen expression in the central nervous system in human immunodeficiency virus encephalopathy. Am J Pathol 164:2089-2099.

Garden GA, Budd SL, Tsai E, Hanson L, Kaul M, D’Emilia DM, Friedlander RM, Yuan J, Masliah E, Lipton SA (2002) Caspase cascades in human immunodeficiency virus-associated neurodegeneration. J Neurosci 22:4015-4024.

Gelbard HA, James HJ, Sharer LR, Perry SW, Saito Y, Kazee AM, Blumberg BM, Epstein LG (1995) Apoptotic neurons in brains from paediatric patients with HIV-1 encephalitis and progressive encephalopathy. Neuropathol Appl Neurobiol 21:208-217.

Giulian D, Vaca K, Noonan CA (1990) Secretion of neurotoxins by mononuclear phagocytes infected with HIV-1. Science 250:1593-1596.

Giulian D, Yu J, Li X, Tom D, Li J, Wendt E, Lin SN, Schwarcz R, Noonan C (1996) Study of receptor-mediated neurotoxins released by HIV-1infected mononuclear phagocytes found in human brain. J Neurosci 16:3139-3153.

Goll DE, Thompson VF, Li H, Wei W, Cong J (2003) The calpain system. Physiol Rev 83:731-801.

Guttmann RP, Baker DL, Seifert KM, Cohen AS, Coulter DA, Lynch DR (2001) Specific proteolysis of the NR2 subunit at multiple sites by calpain. J Neurochem 78:1083-1093.

Heyes MP, Rubinow D, Lane C, Markey SP (1989) Cerebrospinal fluid quinolinic acid concentrations are increased in acquired immune deficiency syndrome. Ann Neurol 26:275-277.

Heyes MP, Ellis RJ, Ryan L, Childers ME, Grant I, Wolfson T, Archibald S, Jernigan TL (2001) Elevated cerebrospinal fluid quinolinic acid levels are associated with region-specific cerebral volume loss in HIV infection. Brain 124:1033-1042.

Jiang ZG, Piggee C, Heyes MP, Murphy C, Quearry B, Bauer M, Zheng J, Gendelman HE, Markey SP (2001) Glutamate is a mediator of neurotoxicity in secretions of activated HIV-1-infected macrophages. J Neuroimmunol 117:97-107.

Kaul M, Garden GA, Lipton SA (2001) Pathways to neuronal injury and apoptosis in HIV-associated dementia. Nature 410:988-994.

Klein RC, Prorok M, Castellino FJ (2003) Direct binding properties of conantokins to native $\mathrm{N}$-methyl-D-aspartate receptors. J Pept Res 61:307-317.

Lipton SA (1993) Human immunodeficiency virus-infected macrophages, gp120, and N-methyl-D-aspartate receptor-mediated neurotoxicity. Ann Neurol 33:227-228.

Lipton SA (2004) Failures and successes of NMDA receptor antagonists: molecular basis for the use of open-channel blockers like memantine in the treatment of acute and chronic neurologic insults. NeuroRx 1:101-110.

Liu L, Wong TP, Pozza MF, Lingenhoehl K, Wang Y, Sheng M, Auberson YP, Wang YT (2004) Role of NMDA receptor subtypes in governing the direction of hippocampal synaptic plasticity. Science 304:1021-1024. 
Lynch DR, Guttmann RP (2001) NMDA receptor pharmacology: perspectives from molecular biology. Curr Drug Targets 2:215-231.

Lynch DR, Guttmann RP (2002) Excitotoxicity: perspectives based on $N$-methyl-D-aspartate receptor subtypes. J Pharmacol Exp Ther 300:717-723.

Masliah E, Ge N, Achim CL, Hansen LA, Wiley CA (1992) Selective neuronal vulnerability in HIV encephalitis. J Neuropathol Exp Neurol 51:585-593.

Meeker RB, Boles JC, Bragg DC, Robertson K, Hall C (2004) Development of neuronal sensitivity to toxins in cerebrospinal fluid from HIV-type 1-infected individuals. AIDS Res Hum Retroviruses 20:1072-1086.

Monyer H, Burnashev N, Laurie DJ, Sakmann B, Seeburg PH (1994) Developmental and regional expression in the rat brain and functional properties of four NMDA receptors. Neuron 12:529-540.

Nottet HS, Flanagan EM, Flanagan CR, Gelbard HA, Gendelman HE, Reinhard JF Jr (1996) The regulation of quinolinic acid in human immunodeficiency virus-infected monocytes. J Neurovirol 2:111-117.

Ohagen A, Ghosh S, He J, Huang K, Chen Y, Yuan M, Osathanondh R, Gartner S, Shi B, Shaw G, Gabuzda D (1999) Apoptosis induced by infection of primary brain cultures with diverse human immunodeficiency virus type 1 isolates: evidence for a role of the envelope. J Virol 73:897-906.

Petito CK, Roberts B (1995) Evidence of apoptotic cell death in HIV encephalitis. Am J Pathol 146:1121-1130.

Petito CK, Roberts B, Cantando JD, Rabinstein A, Duncan R (2001) Hippocampal injury and alterations in neuronal chemokine co-receptor expression in patients with AIDS. J Neuropathol Exp Neurol 60:377-385.

Power C, McArthur JC, Nath A, Wehrly K, Mayne M, Nishio J, Langelier T, Johnson RT, Chesebro B (1998) Neuronal death induced by brainderived human immunodeficiency virus type 1 envelope genes differs between demented and nondemented AIDS patients. J Virol 72:9045-9053.

Ray SK, Hogan EL, Banik NL (2003) Calpain in the pathophysiology of spinal cord injury: neuroprotection with calpain inhibitors. Brain Res Brain Res Rev 42:169-185.

Roberts-Lewis JM, Savage MJ, Marcy VR, Pinsker LR, Siman R (1994) Immunolocalization of calpain I-mediated spectrin degradation to vulnerable neurons in the ischemic gerbil brain. J Neurosci 14:3934-3944.
Sa MJ, Madeira MD, Ruela C, Volk B, Mota-Miranda A, Paula-Barbosa MM (2004) Dendritic changes in the hippocampal formation of AIDS patients: a quantitative Golgi study. Acta Neuropathol (Berl) 107:97-110.

Simpkins KL, Guttmann RP, Dong Y, Chen Z, Sokol S, Neumar RW, Lynch DR (2003) Selective activation induced cleavage of the NR2B subunit by calpain. J Neurosci 23:11322-11331.

Tardieu M, Hery C, Peudenier S, Boespflug O, Montagnier L (1992) Human immunodeficiency virus type 1-infected monocytic cells can destroy human neural cells after cell-to-cell adhesion. Ann Neurol 32:11-17.

Valle M, Price RW, Nilsson A, Heyes M, Verotta D (2004) CSF quinolinic acid levels are determined by local HIV infection: cross-sectional analysis and modelling of dynamics following antiretroviral therapy. Brain 127:1047-1060.

Waxman EA, Lynch DR (2005) N-methyl-D-aspartate receptor subtypes: multiple roles in excitotoxicity and neurological disease. The Neuroscientist 11:37-49.

Wilcox KS, Buchhalter J, Dichter MA (1994) Properties of inhibitory and excitatory synapses between hippocampal neurons in very low density cultures. Synapse 18:128-151.

Wiley CA, Schrier RD, Nelson JA, Lampert PW, Oldstone MB (1986) Cellular localization of human immunodeficiency virus infection within the brains of acquired immune deficiency syndrome patients. Proc Natl Acad Sci USA 83:7089-7093.

Xiong H, Zeng YC, Lewis T, Zheng J, Persidsky Y, Gendelman HE (2000) HIV-1 infected mononuclear phagocyte secretory products affect neuronal physiology leading to cellular demise: relevance for HIV-1-associated dementia. J Neurovirol 6 [Suppl 1]:S14-S23.

Xiong H, McCabe L, Skifter D, Monaghan DT, Gendelman HE (2003) Activation of NR1a/NR2B receptors by monocyte-derived macrophage secretory products: implications for human immunodeficiency virus type one-associated dementia. Neurosci Lett 341:246-250.

Yamashima $\mathrm{T}$ (2004) $\mathrm{Ca}^{2+}$-dependent proteases in ischemic neuronal death: a conserved "calpain-cathepsin cascade" from nematodes to primates. Cell Calcium 36:285-293.

Yiannoutsos CT, Ernst T, Chang L, Lee PL, Richards T, Marra CM, Meyerhoff DJ, Jarvik JG, Kolson D, Schifitto G, Ellis RJ, Swindells S, Simpson DM, Miller EN, Gonzalez RG, Navia BA (2004) Regional patterns of brain metabolites in AIDS dementia complex. NeuroImage 23:928-935. 